\title{
ON THE RELATION BETWEEN SLIDING AND MIGRATION OF SYMMETRICAL TILT GRAIN BOUNDARIES*
}

\author{
D. Wolf, S. R. Phillpot, J. A. Jaszczak, J. M. Rickman and S. Yip ${ }^{\dagger}$
}

\author{
Materials Science Division \\ Argonne National Laboratory
}

Argonne, Mlinois 60439

\begin{abstract}
The submitted manuscript has been authored by a contractor of the U.S. Government under contract No. W-31-109-ENG-38. under contract U.S. Government retains a Accordingly, the U. S. Government retains or reproduce the published form of this contribution, or allow others to do 50 , for U.S. Government purposes.
\end{abstract}

FEBRUARY 1991

* Work supported by the U. S. Department of Energy, BES-Materials Sciences, under Contract W-31-109-Eng-38.

$\dagger$ Permanent address: Department of Nuclear Engineering, Massachusetts Institute of Technology, Cambridge, MA 02139.

\section{DISCLAIMER}

This report was prepared as an account of work sponsored by an agency of the United States Government. Neither the United States Government nor any agency thereof, nor any of their employees, makes any warranty, express or implied, or assumes any legal liability or responsibility for the accuracy, completeness, or usefulness of any information, apparatus, product, or process disclosed, or represents that its use would not infringe privately owned rights. Reference herein to any specific commercial product, process, or service by trade name, trademark, manufacturer, or otherwise does not necessarily constitute or imply its endorsement, recommendation, or favoring by the United States Government or any agency thereof. The views and opinions of authors expressed herein do not necessarily state or reflect those of the United States Government or any agency thereof. 


\title{
ON THE RELATION BETWEEN SLIDING AND MIGRATION OF SYMMETRICAL TILT GRAIN BOUNDARIES
}

\author{
D. Wolf, S. R. Phillpot, J. A. Jaszczak, J. M. Rickman and S. Yip* \\ Materials Science Division \\ Argonne National Laboratory \\ Argonne IL 60439
}

\begin{abstract}
We demonstrate that, based on their unique geometry, the migration of all symmetrical and certain asymmetrical tilt grain boundaries is necessarily accompanied by sliding parallel to the interface. By contrast, for all other types of grain boundaries no crystallographic necessity exists for migration to be coupled with sliding. Except in the case of the coherent (111) twin boundary in the fcc lattice, the coherently-twinned translational configuration is identified as the saddle-point configuration for the migration of the symmetrical tilt boundaries.
\end{abstract}

PACS: $61.70 . \mathrm{Ng}, 66.30 . \mathrm{Lw}$ 


\section{INTRODUCTION}

The idea that the migration of a grain boundary (GB) normal to the boundary plane and its sliding parallel to the boundary plane should be regarded as distinct kinetic processes seems to be intuitively reasonable. In the case of migration it is only necessary that atoms in the immediate vicinity of the boundary move, whereas in the case of sliding all the atoms in one of the adjoining grains have to move collectively. Thus, without considering the detailed atomic structure of the $G B$, it is not immediately obvious that these two processes should be related. Yet, in practice, GB sliding has frequently been observed during GB migration [1]. On the other hand, in some recent instances a remarkable absence of sliding during migration has been noted [2,3].

Existing descriptions of mechanisms of boundary migration basically fall into one of two categories: models which involve "single-atom transfer" [4] or atom shuffling [1,3-5], and those which involve secondary GB dislocations (SGBDs) and boundary steps $[1,5,6]$. In a recent hot-stage in-situ TEM study of bicrystals of gold [2], relatively small boundary displacements were observed which showed geometrical features consistent with the SGBDstep model $[2,6]$. On the other hand, larger displacements were also observed which apparently occurred without the participation of moving dislocations. Since these experiments appear to provide some support for both types of description, it is an open question as to whether there is a single dominant migration mechanism.

It has long been recognized that the atomic structure plays an important role in determining the mechanism of GB migration and the magnitude of the GB mobility [6,7]. Indeed, a consideration of structural details of boundary sliding as modeled by the shearing of a bicrystal of soap bubbles has led to the suggestion that the processes of sliding and migration are coupled and their rates are proportional for high-angle boundaries, since this would allow boundary motions without significant change in structure [8]. This predicted proportionality 
has been observed experimentally [9], and has also been explicitly demonstrated in moleculardynamics simulations of symmetrical tilt bicrystals [10].

In this paper we demonstrate that, within a simple crystallographic model, migration of a symmetrical tilt grain boundary (STGB) may be viewed as a two-step sliding process. This is also true for other types of GBs with only one atom per plane in the unit cell of the GB (such as certain asymmetrical tilt boundaries). Further, as a consequence of the unique unit-cell geometry of STGBs [11], our crystallographic analysis leads to the identification of the coherently-twinned translational configuration of the STGB as the saddle-point configuration for its migration. By contrast, for all other types of boundaries with more than one atom per atomic plane in their planar unit cell, there is no simple geometrical necessity for sliding and migration to be coupled, and a reshuffling of the atoms during $G B$ migration seems to be a requirement [4].

\section{GEOMETRY OF SYMMETRICAL TILT GRAIN BOUNDARIES}

In any Bravais lattice or any crystal lattice with inversion symmetry, the STGB configuration on a given crystallographic plane, characterized by Miller indices (hkl), may be thought of as having been formed by a rotation of one half of a perfect crystal with respect to the other by an angle of $180^{\circ}$ about the normal to the (hkl) plane, denoted by $<h k l>[11]$. This property arises from the inversion symmetry of these lattices $[11,12]$. Such a rotation of the perfect crystal in Fig. 1(a) (in this case about an axis through a point half way between lattice sites in planes $C$ and $D$ ) results in the inversion of the stacking sequence of (hkl) planes on one side of the GB with respect to the other, leading to the well-known fact that all STGBs may be thought of as general twins (see Fig. 1(b)). However, a rigid-body translation, $\mathrm{T}_{\mathrm{opt}}=\left(\mathrm{T}_{\mathrm{x}}, \mathrm{T}_{\mathrm{y}}\right.$ ) parallel to the GB (x-y) plane, such as to minimize the free energy of the bicrystal, usually results in the destruction of the mirror symmetry in Fig. 1(b), leaving some translated - yet still 
inverted - configuration sketched schematically in Fig. 1(c). We will refer to this configuration with the lowest free energy as the ground state, keeping in mind that more than one ground state, with more or less the same GB energy, may, in principle, exist. For the moment we exclude any coherently-twinned configuration (in which, by definition, one lattice plane is shared by the two halves of the bicrystal; i.e., in which the GB plane is an atom plane), as the ground state (see Sec. IV). In practice, in the fcc and bec lattices, for example, only one such coherent-twin configuration is thought to exist (namely on the (111) and (112) planes, respectively).

The fact that in lattices with inversion symmetry all STGBs may be viewed as special $180^{\circ}$ twist boundaries gives rise to some unique geometrical properties of the STGB configuration on a given plane [11]: namely, its planar unit-cell dimensions are identical to those of the perfect-crystal (hkl) plane, and it has only one atom per plane in the unit cell [compare Figs. 1(a) and (c)]. For rotation about $<\mathrm{hkl}>$ by any twist angle other than $180^{\circ} \mathrm{a}$ twist boundary, with a generally much larger planar unit cell and the same (non-inverted) stacking sequence on the two sides of the GB plane, is obtained. In the next section we will illustrate how this unique geometry of the STGB configuration on a given crystallographic plane leads directly to the necessity for STGB migration to take place via sliding.

The number of planes in the repeat stacking sequence, $\mathrm{P}(\mathrm{hkl})$ (sometimes also referred to as the stacking period), is given by $[11,12]$

$$
P(h k l)=\beta(h k l)\left(h^{2}+k^{2}+l^{2}\right),(\beta(h k l)=1 \text { or } 2)
$$

where, as in $x$-ray structure factors, the particular value of $\beta(h k l)$ depends on the specific combination of odd and even Miller indices. For example, in both the fcc and bcc lattices $\beta(111)=\beta(112)=1$, resulting in $P(111)=3$ and $P(112)=6$. For all other choices of (hkl) in these two lattices, $\mathrm{P}(\mathrm{hkl})$ is 10 (for the (210) and (310) planes) or more. According to Eq. (1), 
$\mathrm{P}(\mathrm{hkl})$ increases rapidly with increasing Miller indices, while simultaneously the interplanar lattice spacing, $d(h k l)$, decreases in the well-known manner, proportionally to $\left(h^{2}+k^{2}+l^{2}\right)^{-1 / 2}$.

It follows that on a lattice plane with only one or two planes in the repeat stacking sequence, such as the (100) and (110) planes in the fcc and bcc lattices, with an ... $|A B| A B \mid \ldots$ planar stacking, the STGB and perfect-crystal configurations are identical because a translation of the STGB configuration, ..AB|BA|.., such that $A \rightarrow B$ (and hence $B \rightarrow A$ ) in one half of the bicrystal re-inverts the stacking sequence [11,12]. The simplest non-trivial case in the fcc lattice is therefore the (111) plane, with three planes in the stacking sequence, for which because of the 3 -fold symmetry axis - a rotation by $60^{\circ} \pm k \times 120^{\circ}(k=0, \pm 1, \pm 2, \ldots)$ about the $<111>$ normal produces the well-known coherent (111) twin boundary. As already mentioned, all other STGBs in the two lattices involve GB planes with at least six planes in the repeat stacking sequence.

Because a large planar stacking sequence is notationally cumbersome to manipulate, to illustrate our main point we choose as an example a case in which there are six planes in the stacking sequence, such as for the (112) planes in the fcc lattice (see Fig. 1). The six planes, denoted A, B, C, D, E and F, have identical atomic configurations and are merely displaced relative to each other parallel to the $\mathrm{GB}(\mathrm{x}-\mathrm{y})$ plane by integer multiples of the two-dimensional vector $d=\left(d_{x}, d_{y}\right)$ (see Fig. 1(a)). A translation by $d$ transforms an A plane into a B plane, a B plane into a $\mathrm{C}$ plane, etc. Since there are six planes in the stacking sequence considered here, a translation by $n d(n \leq 6)$ is identical to a translation of $(6-n)$ d. (Notice that the atoms in each of the planes in Fig. 1 are periodically arranged in the $x-y$ plane; the edges of a single planar unit cell are indicated by vertical dashed lines.) On rotation of the bottom half of the ideal crystal in Fig. 1(a) with respect to the top by $180^{\circ}$ to produce the inverted stacking sequence in Fig 1(b), two planes of atoms (here denoted by $F$ ) are moved right on top of each other, resulting in the (unstable) translational configuration with the highest possible GB energy for that plane [11]. Although the optimum rigid-body translation, $\mathrm{T}_{\mathrm{opt}}$, leading to the minimum-energy con- 
figuration, does not necessarily have to be a multiple of $d$, for the purpose of illustration, and with no loss of generality, in Fig. 1(c) we assume this translation to be given by $T_{\text {opt }}=2 \mathrm{~d}$ (or equivalently, $\left.\mathbf{T}_{\text {opt }}=-4 d\right)$.

\section{SLIDING-INDUCED GRAIN-BOUNDARY MIGRATION}

An elementary migration step of a GB requires that the first plane, say, in the lower half of the bicrystal is transformed into the first lattice plane of the upper half, and hence attached in perfect registry. The essence of our argument is as follows: In any GB with only one atom per lattice plane in the planar unit cell on each side of the interface, such a transformation can only take place by a (well-defined) translation parallel to the GB plane. Moreover, since out of infinitely many possibilities only one such translation parallel to the GB leads to the desired transformation, it follows that sliding is a necessary but not sufficient prerequisite of GB migration. For GBs with a larger number of atoms per plane in the unit cell such a transformation by a translation alone is not possible. Instead, a reshuffling or a coherent rotation of the atoms is necessary to bring about such a realignment of a plane from belonging to one half of the bicrystal to the other.

As illustrated in Fig. 2, the migration of any STGB may be broken down into a sequence of two sliding operations, in which the final configuration, Fig. 2(c) is structurally and energetically identical to the initial configuration in Fig. 2(a) (modulo a symmetry operation of the entire system), resulting in a displacement of the GB plane by one interplanar spacing in the direction of the GB normal. Figure 2 illustrates an elementary migration step of the STGB in Fig. 1(c), initially situated between planes $F$ and B, in terms of such a two-step sliding process.

To bring about a downward movement of the GB plane in Fig. 2(a), the first plane in the lower half has to be attached, in perfect registry, to the upper half. The first step, therefore, represents a rigid-body displacement, denoted by $T_{1}$, of the lower half of the bicrystal parallel 
to the GB plane such that the first plane in the lower half of the bicrystal becomes a coherentlytwinned plane in common to both halves. In our example, this highly symmetrical configuration is reached by a translation $T_{1}=-d$, resulting in the $B$ plane at the interface becoming a twinned A plane, which is in perfect registry with both the upper and lower halves of the bicrystal (Fig. 2(b)). (In practice, $\mathbf{T}_{1}$ will of course not be a multiple of $\mathbf{d}$ unless $\mathrm{T}_{\mathrm{opt}}$ is also a multiple of $\mathbf{d}$.) The net effect of this first rigid-body translation is a downward movement of the GB plane by one-half of a lattice plane, transforming the B plane from being nearest to the GB to becoming the GB plane itself). Next, a rigid-body translation of the lower half of the bicrystal by the vector, $T_{2}$, leads to the new ground state in Fig. 2 (c) with the GB having migrated downward by one lattice plane. For STGBs with only a single translational ground state this second rigid-body translation, $T_{2}$, takes the GB from the coherently-twinned configuration in Fig. 2(b) to the new ground state in Fig. 2(c). In our example, a rigid-body translation by $T_{2}=-3 d$ of only those atoms below the twinned A plane in Fig. 2(b) is thus required. This produces the configuration shown in Fig. 2(c), in which the GB plane has now moved downwards by a total of one interplanar spacing relative to Fig. 2(a). That the configuration in Fig. 2(c) is energetically equivalent to that in Fig. 2(a) is easily seen by simply relabeling all lattice planes of the system such that $A \rightarrow F, F \rightarrow E$, etc.; i.e., by applying a translation of $-d$ to the system as a whole (see Fig 2(d)).

In the fcc lattice, with the exception of the (111) plane, the coherently-twinned configuration sketched in Fig. 2(c) has a higher GB energy than the translationally optimized ground state in Fig. 2(a). It may thus be thought of as the saddle point through which the system has to pass during GB migration. In this saddle-point configuration, the twinned plane [plane A in Fig. 2(b)] represents the GB-plane itself. Since this plane is being shared symmetrically by both halves of the bicrystal, the system may subsequently either slide back to the starting configuration (thus aborting an unsuccessful attempt to migrate), or it can slide on, by a vector $T_{2}$, into a new ground state. In the first scenario, following an unsuccessful 
excursion to the saddle point the twinned A plane re-attaches itself to the lower half, while in a successful migration attempt it ultimately attaches itself to the upper half.

Given that a rigid-body translation represents generally ihree (so-called microscopic) degrees of freedom (DOFs), one can expect the two-dimensional in-plane translations $T_{1}$ and $T_{2}$ to be accompanied by a local volume change at the GB (i.e., a translation perpendicular to the GB). In fact, since in metals the GB energy is approximately proportional to the volume increase per unit area at the interface [13], one would expect any attempt of the GB to slide towards the saddle point to be accompanied by a volume increase. 


\section{DISCUSSION}

The above interpretation of GB migration as a two-step sliding process, with the twinned configuration as the saddle-point configuration, can obviously not be operative in an STGB in which the coherently-twinned configuration is the ground state. For example, the STGB on the (111) plane in the fcc lattice [with $P(111)=3$ ], in which $T_{\text {opt }}$ produces the wellknown (111) twin boundary, ...CABICAB C BAClBACl... (with the $\mathrm{C}$ plane twinned in this example), represents a special case in which the ground state and the coherently-twinned configuration are identical. Therefore, at first sight one would expect that no activation barrier has to be overcome to induce GB migration. However, for any twinned ground-state configuration a simple one-step translation can be chosen which results in the displacement of the GB plane by one full interplanar spacing. In the case of the (111) twin in the fcc lattice, such a translation is one in which $B \rightarrow A$ in the lower half of the bicrystal, resulting in the configuration ...ICABC A CBI... (in which the A plane is now twinned). Since for any translation away from the low-energy twinned configuration the energy increases dramatically, this process is likely to involve a very high energy barrier to sliding, in accord with the wellknown low-mobility of this interface. Furthermore, a simple crystallographic argument as to which translation leads to the saddle-point configuration cannot be made in this case.

Although from the above it is clear that in the fcc lattice the migration of all STGBs except the (111) twin may be viewed as due to two well-defined sliding operations, $T_{1}$ and $T_{2}$, it is also clear that GB sliding by some arbitrary vector $T$ does not necessarily result in GB migration [8], as illustrated in Fig. 3. Starting from the ground state of Fig. 1(c) (see Fig. 3(a)), the lower half of the bicrystal may slide, for example, by $T=2 d$ to the new configuration in Fig. 3(b). That this configuration has the same energy as the ground state in Fig. 3(a) is again seen by simply relabeling all lattice planes of the entire system, such that $A \rightarrow C, B \rightarrow D$, etc.; i.e., by applying a translation of $2 d$ to the system as a whole. The resulting configuration, Fig. 3(c), is identical to Fig. 3(a), however with the entire bicrystal 
turned upside down. Therefore this sliding between two isoenergetic states takes place with no GB migration.

Since only one (or, for STGBs with more than one ground state, only a few) crystallographically well-defined translations lead to the saddle point for GB migration, one would expect that the application of an external shear stress leads to GB migration only under well-defined crystallographic circumstances. Also, as a consequence of the above interpretation of GB migration as a two-step sliding process, with the twinned configuration as the saddle-point configuration, the activation energy for GB migration should be governed by the related difference in energy between the ground state and the coherently-twinned configuration. However, in STGBs with more than one translational ground state, $\mathrm{T}_{\mathrm{opt}}$, a distribution of saddle-point energies, and of the corresponding values of $T_{1}$ and $T_{2}$, is expected.

The above arguments are, of course, purely crystallographic by nature, valid for all GBs with only one atom per lattice plane in the unit cell. Although in the above discussion only symmetrical tilt boundaries were considered, there exist many asymmetrical tilt boundaries (ATGBs) which also have only one atom per plane in the unit cell on each side of the interface, and for which the above geometrical considerations should apply equally. The only difference is that the saddle-point configuration due to the asymmetry in the GB plane cannot be a twinned state. However, the rigid-body translation $\mathrm{T}_{1}$ is still well defined in this case as that vector which attaches the uppermost plane of the lower crystal in perfect registry to the upper crystal. Again, the reasons why such a simple migration mechanism might be operative even in this special group of asymmetrical GBs lie in their unique unit-cell geometry. With only one atom per lattice plane on both sides of the interface, a translation parallel to the GB plane is sufficient to transform a lattice plane from belonging to the bottom half to one in the top crystal.

In all other GBs, a simple rigid-body translation cannot bring about such a realignment of a lattice plane, and a reshuffling of atoms is required for the GB to migrate; i.e., there is no 
purely crystallographic reason why such boundaries, with a larger number of atoms in the planar unit cell, should slide during migration. Instead, their migration involves cooperative rotations and realignments of all the atoms in an atomic plane. However, since sliding accompanied by a local volume expansion provides three extra degrees of freedom which can be energetically optimized throughout the migration process, it is possible in practice that migration accompanied by sliding may result in a lower energy barrier than migration without sliding [8]. We mention, however, that in our recent molecular-dynamics investigation of the migration of a twist boundary no such effects were observed [3].

Of course, one should keep in mind that the above purely crystallographic analysis has not included any effects arising, for example, from point defects in the GB region, such as impurity segregation and reconstruction or the Frenkel-like defects observed in our simulations [3], and that the idealized grain boundaries described here may or may not exist in this form in nature.

In conclusion, we have illustrated how, for simple crystallographic reasons, in all symmetrical tilt boundaries as well as those asymmetrical tilt boundaries with only one atom per plane in the unit cell, migration normal to the interface plane is inevitably accompanied by sliding parallel to the interface plane. This unique migration mechanism thus seems to be closely connected with the unique geometry of these GBs. In particular, their migration involves only the three microscopic degrees of freedom associated with the relative translation of the two grains. By contrast, in all other types of GBs a complex many-body reshuffling of the atoms at the GB must take place in order to realign a lattice plane with the semicrystal on the opposite side of the GB. Any observed sliding associated with their migration therefore arises from the energetics and dynamics of this reshuffling rather than from crystallographic necessity.

\section{ACKNOWLEDGMENTS}


We gratefully acknowledge helpful discussions with D. A. Smith. This work was supported by the U.S. Department of Energy, BES Materials Sciences, under Contract W-31-109-Eng-38. 


\section{REFERENCES}

* Permanent address: Department of Nuclear Engineering, Massachusetts Institute of Technology, Cambridge MA 02139.

1. See, for example, H. Gleiter and B. Chalmers, Progr. Mater. Sci. 16, 127 (1972).

2. S. E. Babcock and R. W. Balluffi, Acta Metall. 37, 2357 (1989); ibid. 2367 (1989).

3. J. M. Rickman, S. R. Phillpot, D. Wolf, D. L. Woodraska and S. Yip, J. Mater. Res. (submitted; preceding paper); see also J. M. Rickman, S. R. Phillpot and D. Wolf, MRS Symposium Proceedings 193, 325 (1990).

4. C. L. Bauer, J. Physique 43, Suppl. 12, C6-187 (1982).

5. H. Gleiter, Acta Metall. 17, 565 (1969); ibid. 853 (1969).

6. D. A. Smith, C. M. F. Rae and C. R. M. Grovenor, in Grain-Boundary Structure and Kinetics, ASM, Metals Park, 1980, p. 337.

7. D. A. Smith, Ultramicroscopy 29, 1 (1989).

8. M. F. Ashby, Surf. Sci. 31, 498 (1972).

9. J. L. Walter and H. E. Cline, Trans. Met. Soc. AIME 242, 1823 (1968).

10. G. H. Bishop, R. J. Harrison, T. Kwok and S. Yip, J. Appl. Phys. 53, 5596 (1982).

11. D. Wolf, J. Phys. Colloq. 46, C4-197 (1985).

12. D. Wolf and J. F. Lutsko, Z. Kristallographie 189, 239 (1989).

13. D. Wolf, Scripta Metall. 23, 1913 (1989). 


\section{FIGURE CAPTIONS}

FIG. 1. Generation of a symmetrical tilt boundary on the (hkl) plane of a Bravais lattice by a $180^{\circ}$ twist rotation about the plane normal, <hkl> (schematic) [11]. (a) A crystal oriented normal to some (hkl) plane which, in this example, is taken to have six planes in the repeat stacking sequence (such as for (112) planes in the fcc or bcc lattice). Here $\mathrm{d}$ is the two-dimensional translation vector which transforms, for example, an A plane to a $\mathrm{B}$ plane. (b) $\mathrm{A} 180^{\circ}$ rotation, in this case about a point half way between lattice sites in planes $C$ and $D$, is applied to the lower half of the perfect crystal with respect to the top half, with the result that the stacking sequence is reversed. This configuration, with two identical planes on top of one another, has the highest energy of any translational configuration and is therefore usually unstable [11]. Here and throughout, the GB plane is denoted by a horizontal bold line. (c) A rigid-body translation of the lower half with respect to the upper by a vector $T_{\text {opt }}$ (here taken to be $T_{\text {opt }}=2 d$ ) minimizes the GB energy and results in the ground-state configuration of the STGB on the (hkl) plane. In practice, $T_{\text {opt }}$ need not be a multiple of $d$.

FIG. 2. Two-step sliding process leading to STGB migration (schematic). (a) Ground-state configuration of Fig. 1(c) from which sliding and migration are considered. (b) Sliding of the lower half of the STGB in (a) by $\mathbf{T}_{1}$ (=-d in this case) produces a twinned plane, labeled A, shared by the two halves of the bicrystal. (c) Sliding of all the planes below the twinned A plane by $T_{2}(=-3 \mathrm{~d}$ in this case) results in a completed migration event of the $\mathrm{GB}$ by one interplanar spacing, $\mathrm{d}(\mathrm{hkl})$, relative to the initial configuration in (a). (d) That the configuration in (c) is, indeed, identical to that in (a) is easily seen by simply relabeling all lattice planes of the entire system such that $A \rightarrow F, E \rightarrow D$, etc.; i.e., by applying a translation of $-d$ to the system as a whole. 
FIG. 3. For $T \neq T_{1}, T \neq T_{2}$ GB sliding takes place without migration (schematic). (a) Groundstate configuration of Fig. 1(c) from which sliding is considered. (b) Sliding of the lower half by $T=2 d(\neq T 1$ ) does not result in $G B$ migration. (c) By relabeling all lattice planes of the system, such that $A \rightarrow C, B \rightarrow D$, etc.; i.e., by applying a translation of $2 \mathbf{d}$ to the system as a whole, it is seen that the configurations (a) and (b) are isoenergetic. 


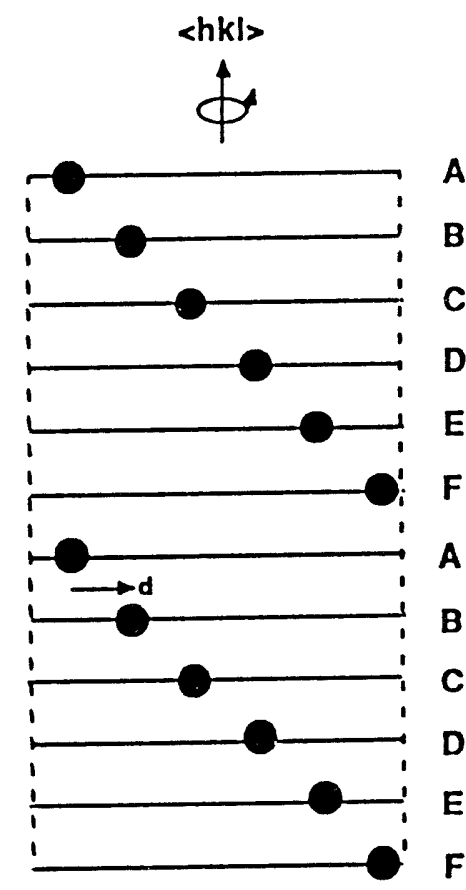

(a)
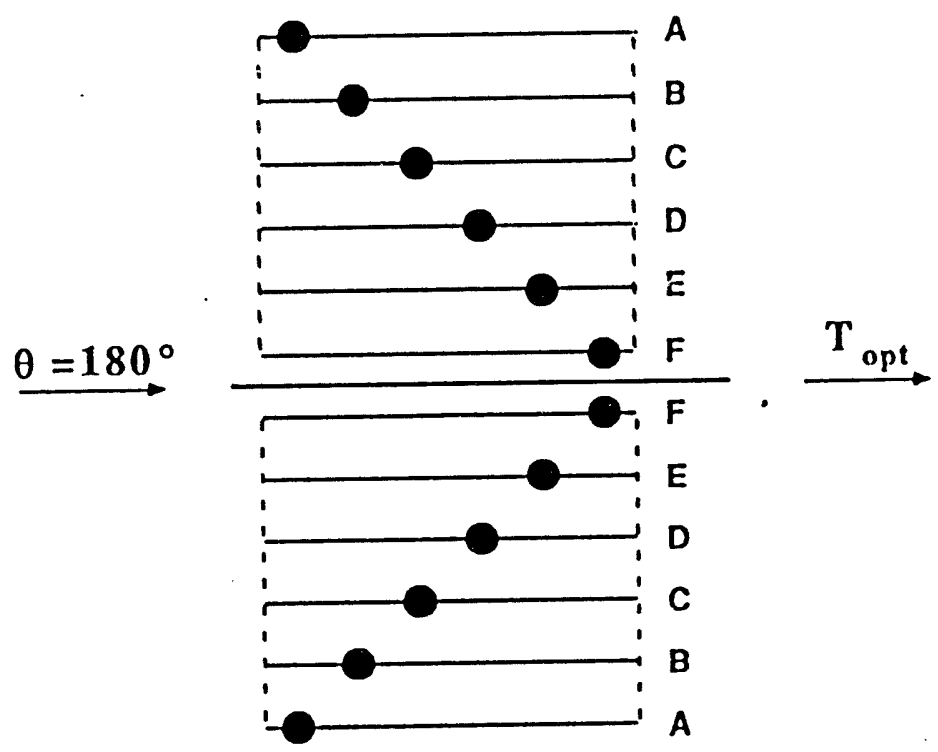

(b)

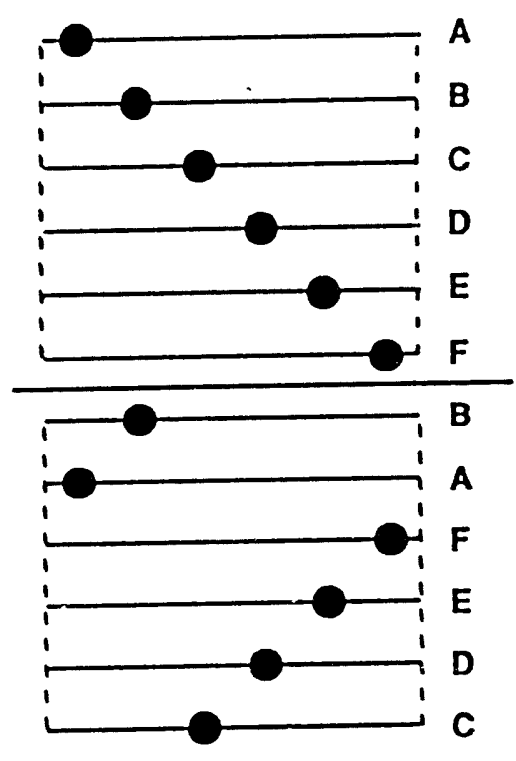

(c) 
(p)

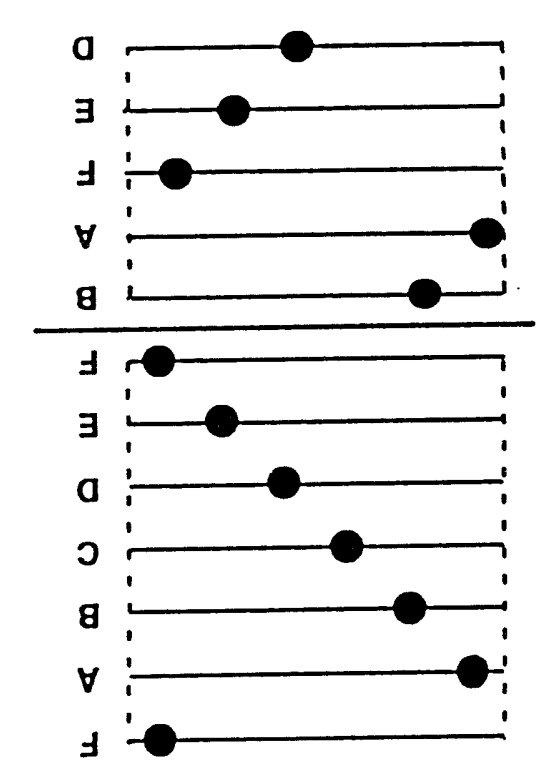

(0)

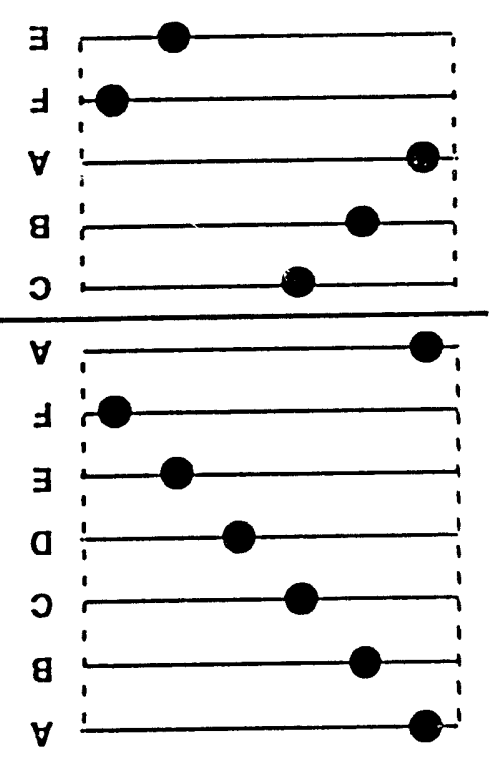

(9)

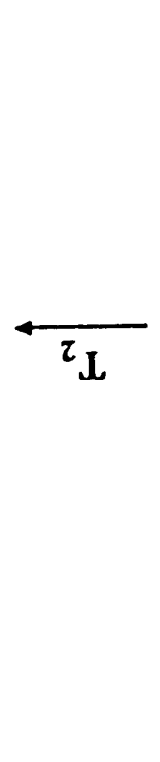

(e)

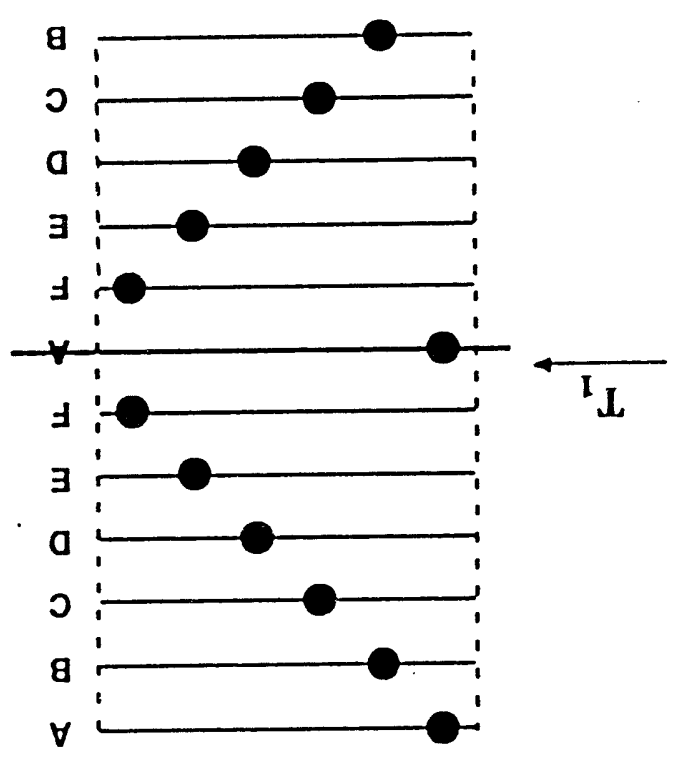

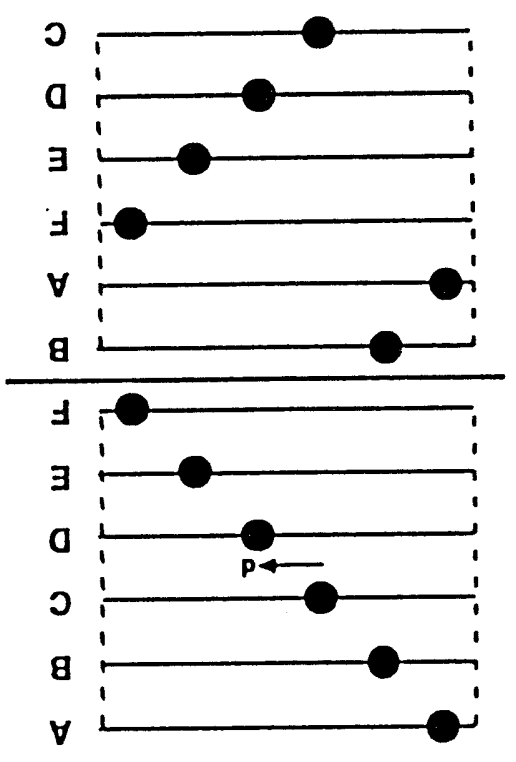




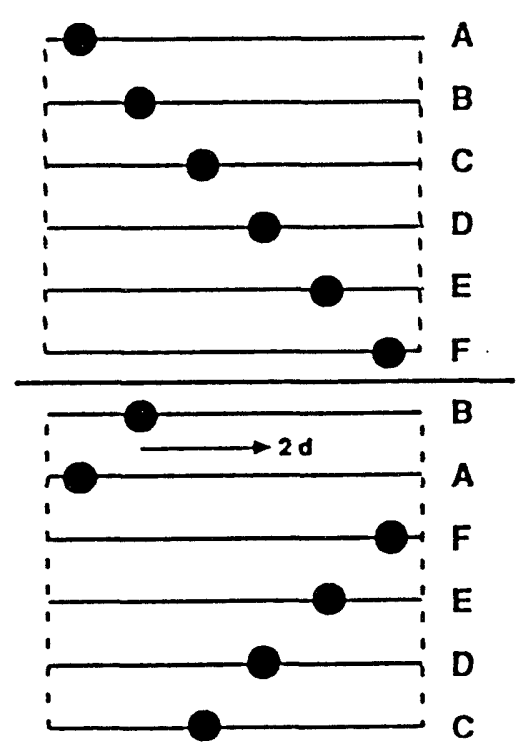

(a)
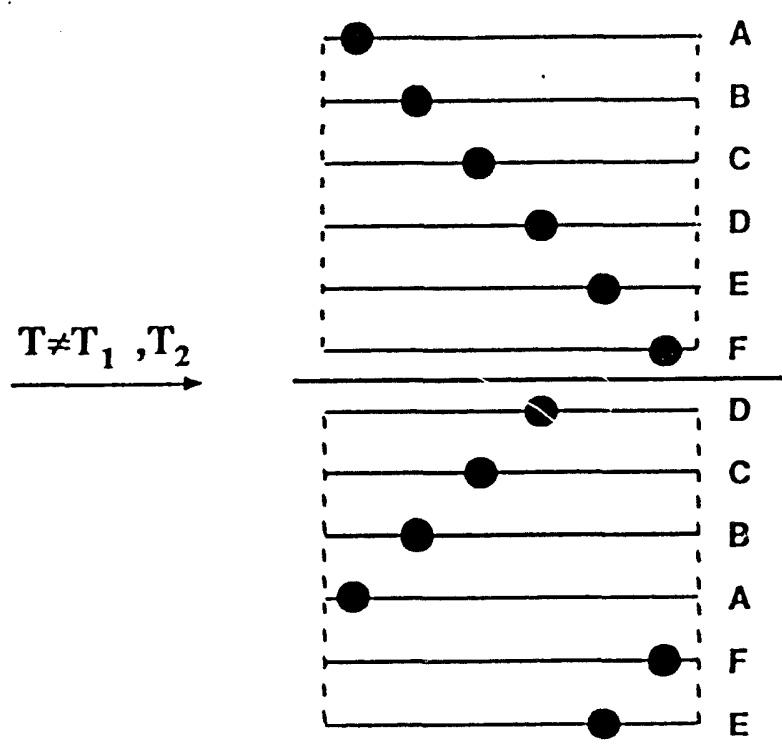

(b)

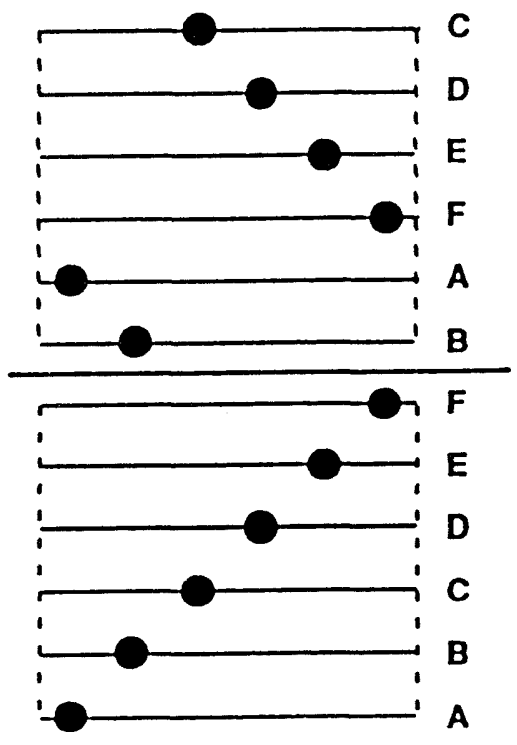

(c) 


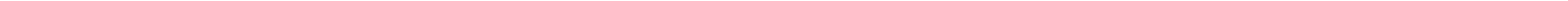




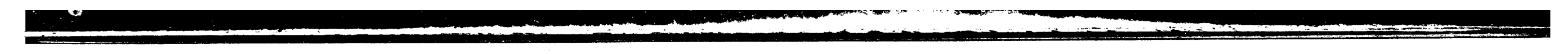

